Simon Parent

Université de Montréal

(Canada)

\section{Éthier, M.-A., Lefrançois, D. et Joly-Lavoie, A. (dir.). (2018). \\ Mondes profanes: Enseignement,
fiction et histoire. Québec, QC: Presses
de I'Université Laval. Mondes profanes: Enseignement,
fiction et histoire. Québec, QC : Presses
de l'Université Laval. Mondes profanes: Enseignement,
fiction et histoire. Québec, QC : Presses
de l'Université Laval.}

doi:10.18162/fp.2019.a169

\title{
RECENSION
}

Mondes profanes est un ouvrage collectif où s'imposent les contrastes et dont la pertinence s'ancre profondément dans la société contemporaine, que ce soit dans le domaine de la recherche en sciences humaines ou dans une perspective d'enseignement de l'histoire en contexte scolaire. L'objectif principal se décline d'ailleurs selon ces deux pendants: d'une part, les auteurs souhaitent diffuser les connaissances et opinions relatives aux discours profanes de l'histoire, et d'autre part, en tracer les différentes utilisations potentielles dans les salles de classe du Québec et d'ailleurs.

Afin de mieux comprendre ces notions de discours et d'histoire profanes, les auteurs nous introduisent à un clivage existant entre deux perspectives : l'histoire qualifiée de savante, issue de l'étude scientifique du récit historique, et l'histoire profane, véhiculée par un discours public qui trace un portrait simplifié, voire altéré, du récit historique. Afin de favoriser la progression du lecteur, cet ouvrage a été organisé en tenant compte de cette distinction qui peut d'emblée paraître subtile. Ainsi, les deux premiers chapitres jettent les bases conceptuelles et théoriques de l'histoire savante et profane, puis les chapitres ultérieurs abordent différentes réflexions contextualisées et organisées en quatre catégories: arts de la scène (télévision, cinéma et théâtre); jeux vidéos (construction, stratégie et aventure); enseignement de l'histoire, fiction et narration (récit historique et enquête historienne); et culture publique (artéfact, lieu, musée et reconstitution). Les 37 auteurs ayant collaboré à l'écriture de cet ouvrage possèdent des 
expériences professionnelles variées (chercheurs, enseignants, conseillers pédagogiques, etc.), permettant ainsi d'assurer une complémentarité qui caractérise la collaboration entre le milieu scientifique et les milieux de pratique. On se réjouit d'ailleurs de remarquer l'articulation constante des volets scientifique (recension d'écrits) et pratique (étude de cas et scénario pédagogique), dans chacun des 14 chapitres.

Dans la section Histoire et arts de la scène, on prend d'abord la mesure du phénomène télévisuel, qui possède un rôle de premier plan dans la construction des mémoires collectives. Alors que certains attribuent à ce type de discours l'épithète "simplifié ", d'autres y voient plutôt une occasion de mobiliser des habiletés intellectuelles et la rigueur. Il en va de même pour les images médiatiques, qui sont très propices à la mobilisation de la pensée critique : les auteurs présentent d'ailleurs des exemples fortement évocateurs, comme la comparaison d'images originales et altérées de dirigeants de l'Union soviétique. On voit dès lors naître une préoccupation pour la pensée critique, qui est d'ailleurs évoquée et déclinée de plusieurs façons dans chacun des chapitres, en plus d'être souvent associée (voire intégrée) à la pensée historienne. Le chapitre sur le cinéma et le théâtre n'y fait pas exception, alors qu'on réitère l'importance d'éduquer les jeunes aux médias, surtout en considérant que les sixième et septième arts seront sans doute appelés à évoluer au gré des innovations technologiques à venir.

Puis, dans la section Histoire et jeux vidéos, le lecteur est invité à découvrir des univers virtuels qui représentent des sources riches et fécondes d'opportunités d'étudier l'histoire, et ce, malgré de nombreux a priori négatifs issus d'une questionnable opposition entre le caractère sérieux de l'histoire et l'allure anodine du jeu. Ce discours profane permet une forme d'action particulièrement puissante et engageante au regard de l'apprentissage de concepts historiques et géographiques, que ce soit en construisant des maquettes historiques dans le monde virtuel du jeu Minecraft, ou encore en jouant à des jeux vidéos d'histoire comme Assassin's Creed, où l'élève est appelé à prendre des décisions et à intervenir dans des événements du passé. Or, les auteurs rappellent que la réflexivité doit impérativement être au cœur des préoccupations de l'enseignant, qui peut par exemple amener l'élève à s'exprimer pendant des séances de jeu pour verbaliser sa compréhension.

Vient ensuite la section Enseignement de l'histoire, fiction et narration, où il est question du roman historique, du roman graphique et de la chanson. Alors que le premier aurait la capacité de faciliter la compréhension de réalités historiques complexes et de sensibiliser les apprenants au présentisme, le second (roman graphique) combine l'histoire savante et profane en une approche dialectique propice au développement d'habiletés comme le scepticisme sain et la vigilance à l'égard de la pensée réductrice. La troisième (la chanson) s'ancre distinctement dans la culture populaire, considérant le rôle d'expression qu'elle a joué chez les individus et les groupes marginalisés dans l'histoire. En plus de favoriser l'empathie, elle peut être utilisée à la fois comme source historique et comme amorce d'une activité d'apprentissage.

Enfin, dans la section Histoire et culture publique, les auteurs déclinent les nombreux rôles assumés par les musées, outre leur mission primaire d'assurer l'accès à des artéfacts. En effet, ces institutions permettent non seulement la genèse de réflexions critiques et la mobilisation de la pensée historienne, mais elles permettent aussi d'affecter positivement les attitudes des apprenants envers l'histoire, en tant que matière scolaire, et les musées, lieux d'éducation non formelle. Les auteurs évoquent aussi les reconstitutions historiques qui, malgré leur difficulté à mobiliser la pensée historienne, permettent une appropriation du passé par les apprenants et un engagement notable. Le dernier chapitre aborde le 
patrimoine bâti et la culture immatérielle, qui représentent sans doute dans cet ouvrage la manifestation la plus évocatrice de l'intérêt pour la démocratisation de l'étude de l'histoire. En effet, on suggère ici que l'environnement immédiat de l'apprenant peut être, en soi, une partie intégrante de l'histoire profane. On valorise donc ici l'idée selon laquelle l'histoire n'est pas « un ensemble immuable de faits objectifs et étanches à l'interprétation » et qu'elle peut être « actualisée dans un répertoire indéfini de situations ».

Cet ouvrage témoigne d'un souci constant et pertinent de briser les barrières imaginaires que semblent s'imposer plusieurs apprenants et qui entravent l'accès à l'interprétation de l'histoire. L'enseignant s'avère être un acteur-clé pour accompagner les élèves dans leur découverte critique, réflexive et autonome de celle-ci. Finalement, on ne saurait omettre l'intérêt omniprésent dont témoignent les auteurs pour la valorisation de l'histoire profane qui, à défaut d'être valide scientifiquement, peut contribuer de façon significative (tant par éloquence que par importance) à la compréhension du récit historique à l'école.

\section{Pour citer cet article}

Parent, S. (2019). Éthier, M.-A., Lefrançois, D. et Joly-Lavoie, A. (dir.). (2018). Mondes profanes : Enseignement, fiction et histoire. Québec, QC : Presses de l'Université Laval. Formation et profession, 27(1), 134-136.

http://dx.doi.org/10.18162/fp.2019.169 